\title{
A generalized formulation for high resolution spectrometer fringes using the case of a short pulse
}

Chandrasekhar Roychoudhuri, Colin Kelley, Peter Poulos

Chandrasekhar Roychoudhuri, Colin Kelley, Peter Poulos, "A generalized formulation for high resolution spectrometer fringes using the case of a short pulse," Proc. SPIE 9664, Ninth International Topical Meeting on Education and Training in Optics and Photonics, 96640G (24 October 2005); doi: 10.1117/12.2207776 
Ref ETOP091

\title{
A generalized formulation for high resolution spectrometer fringes using the case of a short pulse
}

\author{
Chandrasekhar Roychoudhuri ${ }^{\mathrm{a}}$, Colin Kelley ${ }^{\mathrm{a}}$ and Peter Poulos ${ }^{\mathrm{b}}$
}

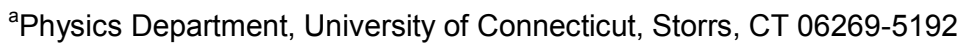

${ }^{b}$ Engineering Department, Manchester Community College, Manchester, CT

\section{Introduction:}

\section{Summary}

Historically, spectrometers are presented as: (i) gratings under diffraction chapter, (ii) Michelson's Fourier transform spectrometry under the two beam interferometry and (iii) Fabry-Perot spectrometry under multiple beam interferometry. We present a unified geometrical and mathematical presentation of all the three major, high resolution, spectrometers that facilitate the learning process and the retention of the physics behind spectrometry.

We also have another deeper motivation behind this paper. Conservation of energy demands that EM signals produced by any physical system will always be of finite duration both in space and in time. They could neither be infinite nor be point-like. Similarly, all natural devices have finite response times and they do not wait and study the signal for the entire duration of the signal to pass through them before reacting to it. Photo sensitive molecules and photo detectors respond to the optical signals in the sub-pico second domains. Thus, we have a built-in conceptual contradiction in emulating nature's operational principles when we use time-frequency Fourier theorem to eliminate the time parameter by accepting the presence of time-infinite signals of many frequencies. This integral is a non-causal integral by definition because a physical entity must wait to read the entire time-infinite signal first. Although, higher level mathematics $[\mathrm{x}]$ attempts to skirt the problem by various mathematical manipulations, the fundamental physical concepts remain confusing in the minds of the young learners. So, in this paper, we develop a method for the direct time-domain propagation for a simple pulse of width $\delta t$ and duration $a(t)$, a(t)exp[-i2mvt], with a carrier frequency $v$, to be determined by spectrometry. The strength of our proposed direct time domain approach is demonstrated by the success (i) in unifying the formulation for apparently three different spectrometers, (ii) in recovering the traditional Fourier frequency interpretation when one integrates our time-evolving fringe expression over a very long period, as we used to do in early days with photographic plates before the advent of very fast photo detectors and electronics, and (iii) in predicting that the de-convolution of our pulse response function from the broadened fringe pattern can help recover the actual carrier frequency of the signal. The last statement is equivalent to claiming that $\delta v_{f} \delta t>1$, which naturally derives from the time-frequency Fourier integral, is not a fundamental limit in classical instrumental spectroscopy. Just as the CW response function (CW-RP) is recognized as an instrumental width, and is de-convolved from the complex CW apparent spectrum to find the actual carrier frequency distribution, so should we de-convolve the pulse response function (derived in this paper) from the recorded apparent pulse spectrum. 


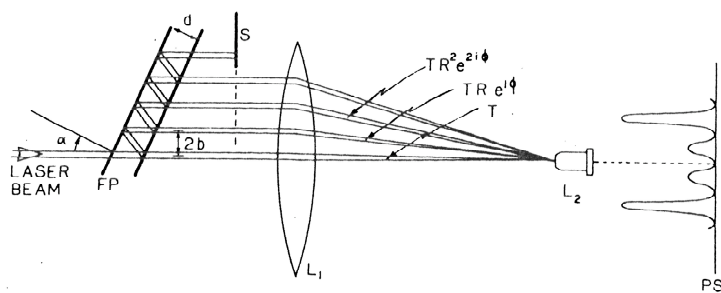

Time domain analysis of spectrometers:

Let us consider classical spectrometers like gratings and Fabry-Perots (FP) with pulsed light [1-5]. They replicate the incident pulse into $N$ partially superposed, delayed pulses, as depicted below in Figs. 1 \& 2. $\mathrm{N}$ is the number of steps for a grating and the reflective finesse for an FP. The time evolving fringe width can be computed by simply taking the square modulus of the partially superposed amplitude train. The apparent "spectral fringe width", is given by the time integration over the duration of the pulse train after taking the square modulus of the sum of all the amplitudes of the partially superposed pulses [5]:
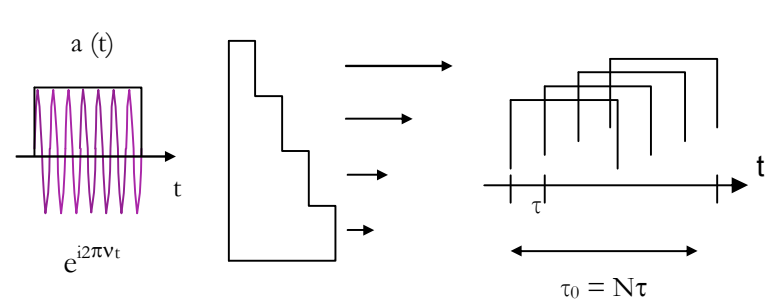

$$
\begin{aligned}
& \text { Figure 2. Partial } \\
& \text { superposition of a train of } \\
& \text { finite pulses with a periodic } \\
& \text { step delay of } \tau \text { produced by } \\
& \text { an echelette grating. The } \\
& \text { carrier frequency, } \nu \text {, of the E- } \\
& \text { vector and the time-finite } \\
& \text { duration of the input } \\
& \text { amplitude, a }(\mathrm{t}), \text { is depicted on } \\
& \text { the left. Notice that all } \\
& \text { spectrometers have a } \\
& \text { characteristic time constant } \\
& \text { for its fringe evolution, or } \\
& \text { pulse stretching, given by }
\end{aligned}
$$

$$
\begin{aligned}
I_{p l s}(v, \tau)=\sum_{n=0}^{N-1} T^{2} R^{2 n} & +2 \sum_{n \neq m}^{N-1} T^{2} R^{n+m} \gamma(|n-m| \tau) \cos [2 \pi(n-m) v \tau] \text {, for an FP. } \\
& =\frac{1}{N}+\frac{2}{N^{2}} \sum_{p=1}^{N-1}(N-p) \gamma(p \tau) \cos [2 \pi p v \tau] \text {, for a grating. }
\end{aligned}
$$

$=\frac{1}{2}+\frac{1}{2} \gamma(p \tau) \cos [2 \pi p v \tau]$, for a Michelson

Where, $\gamma_{\mathrm{nm}}(\tau)$ is the normalized autocorrelation between the $\mathrm{m}$-th and the $\mathrm{n}$-th pulses:

(4)

$$
\gamma(p \tau) \equiv \gamma_{n m}(\tau) \equiv \gamma(|n-m| \tau)=\int a(t-n \tau) a(t-m \tau) d t / \int a^{2}(t) d t
$$

As the width of the pulse exceeds the spectrometer time constant, $\mathrm{T}_{0}$ [2], pulse-response function of Eq. 3 converges to the standard $\mathrm{CW}$ instrumental response curve, $\mathrm{I}_{\mathrm{cw}}(\mathrm{V}, \mathrm{T})$ :

$$
\underset{\delta t \rightarrow \tau_{0}=N \tau}{L t}\left[I_{p l s}(v, \tau)\right]=I_{c w}(v, \tau)
$$

We have thus established the conceptual continuity for the fringe width between those produced by a short pulse and a very long pulse, because $\gamma(p \tau) \rightarrow 1$ as the pulse width, $\delta t \rightarrow \tau_{0}=N \tau$, underscoring the significance of $\tau_{0}$, defined by us as the spectrometer time constant [2]. The beauty of this paper is in the Eq.5, which implies that for all the cases of spectrometers, Eq.3, 3a \& 3b, the traditional CW derivation of text books can be recovered with the help Eq.5. Further, one can also show mathematically, using Parseval's theorem of energy conservation, that the time broadened fringe width can be expressed as the convolution of the CW instrumental response function, $\mathrm{I}_{\mathrm{cw}}(\mathrm{V}, \mathrm{T})$, with the Fourier intensity 
"spectrum", $\tilde{A}(v)$; it is the square modulus of the Fourier transform of the amplitude envelope of the pulse, $a(t)[3,4]$ :

Or, $\quad I_{p l s}(v, \tau)=I_{c w}(v) \otimes \tilde{A}(v)$

This identity relation is very significant because the traditional mistake of taking Fourier frequency as reality turns out to be correct, as far as the fringe width measurement is concerned. However, the direct time domain analysis in Eq.3 shows that only the carrier frequency, $v$, plays the physically important role of determining the location of the fringe $(\mathrm{m}$ $=\mathrm{VT}$ ), and the fringe width is the artifact of time varying amplitude of the pulse. We will also present details of pictorial and conceptual similarity between various spectrometers.

\section{Acknowledgement}

The author would like to acknowledge partial support from Nipon Sheet Glass Co. for related research.

\section{References}

1. A. Kastler, Nouv.Rev. Opt. 5, 133-139 (1974).

2. C. Roychoudhuri, J. Opt. Soc. Am.; 65, 1418 (1976); Boletin Inst.Tonantzintla, 2 (2), 101 (1976).

3. C. Roychoudhuri, D. Lee, Y. Jiang, S. Kittaka, M. Nara, V. Serikov and M. Oikawa, Proc. SPIE Proc.Vol.5246, pp.333-344, (2003).

4. C. Roychoudhuri, SPIE Proc. Vol. 5531, paper \# 60, Annual Conference, 2004.

5. C. Roychoudhuri; Am. J. Phys. 43 (12), 1054 (1975). 